Pesq. Vet. Bras. 37(1):52-57, janeiro 2017

DOI: $10.1590 / \mathrm{S} 0100-736 \mathrm{X} 2017000100009$

\title{
Detecção de proteínas imunorreativas de Rickettsia sp. cepa Mata Atlântica ${ }^{1}$
}

\author{
Caroline S. Oliveira ${ }^{2 *}$, Patricia Bräunig ${ }^{2}$, Felipe Krawczak ${ }^{3}$, Marcelo B. Labruna ${ }^{3}$, \\ Sonia A. Botton ${ }^{2}$, Fernanda S.F. Vogel ${ }^{2}$ e Luis A. Sangioni ${ }^{2 *}$
}

\begin{abstract}
Oliveira C.S., Braunig P., Krawczak F., Labruna M.B., Botton S.A., Vogel F.S.F. \& Sangioni L.A. 2017. [Detection of immunoreactive proteins of Rickettsia sp. Atlantic Forest strain.] Detecção de proteínas imunorreativas de Rickettsia sp. cepa Mata Atlântica. Pesquisa Veterinária Brasileira 37(1):52-57. Departamento de Medicina Veterinária Preventiva, Centro de Ciências Rurais, Universidade Federal de Santa Maria, Av. Roraima 1000, Prédio 44, Sala 5149, Santa Maria, RS 97105-900, Brazil. E-mail: carolsobotyk@gmail.com

Brazilian Spotted Fever (BSF) is an infectious disease transmitted by ticks to humans. A new human rickettsial infection was reported to cause spotted fever in the State of São Paulo and was named Rickettsia sp. Strain Atlantic Forest. This study aimed to detect and identify proteins with potential to stimulate the immune system of mammalian host of this new strain described. Therefore, we performed total protein extraction Rickettsia sp. Strain Atlantic Forest. The extracted proteins were fractionated by electrophoresis. The protein bands were transferred to nitrocellulose membrane by electrical migration and subjected to Western blot for protein detection. In all, seven immunoreactive proteins were detected. Two proteins showed higher abundance, with molecular weight of 200 and $130 \mathrm{kDa}$ respectively. By comparing existing proteomic maps and the molecular weight of these proteins showed that, it is suggested that the two proteins detected representing rOmpA (200 $\mathrm{kDa})$ and $\mathrm{rOmpB}(130 \mathrm{kDa})$. The other detected proteins had lower occurrence and molecular weight less than $78 \mathrm{kDa}$, which may represent members of the cell surface antigens Family (Sca-Surface cell antigen). The detected proteins may serve as a study based on the development of sensitive and specific diagnostic methods in the development of vaccines and they enable further studies to more effective therapies.
\end{abstract}

INDEX TERMS: Immunoreactive proteins, Rickettsia sp. Atlantic Forest strain, rickettsial diseases, intracellular bacteria, Brazilian Spotted Fever, immunogenic proteins.

RESUMO.- A Febre Maculosa Brasileira (FMB) é uma doença infecciosa, transmitida por carrapatos ao homem. Uma nova riquetsiose humana foi descrita como causadora de Febre Maculosa no Estado de São Paulo, sendo denominada de Rickettsia sp. cepa Mata Atlântica. O presente trabalho teve como objetivo detectar e identificar proteínas com potencial de estimular o sistema imune de hospedeiro mamí-

\footnotetext{
${ }^{1}$ Recebido em 22 de março de 2016.

Aceito para publicação em 9 de junho de 2016.

${ }^{2}$ Departamento de Medicina Veterinária Preventiva (DMVP), Centro de Ciências Rurais (CCR), Universidade Federal de Santa Maria (UFSM), Avenida Roraima 1000, Prédio 44, Sala 5149, Santa Maria, RS 97105-900, Brasil. *Autor para correspondência: carolsobotyk@gmail.com

${ }^{3}$ Departamento de Medicina Veterinária Preventiva e Saúde Animal, Faculdade de Medicina Veterinária e Zootecnia (FMVZ), Universidade de São Paulo (USP), Avenida Prof. Orlando Marques de Paiva 87, São Paulo, SP 05508-270, Brasil.
}

fero, desta nova cepa descrita. Para tanto, foi realizado a extração proteica total de Rickettsia sp. cepa Mata Atlântica. As proteínas extraídas foram fracionadas por eletroforese. As bandas proteicas foram transferidas para membranas de nitrocelulose por migração elétrica e submetidas à técnica de Western-blot, para detecção proteica. Ao todo sete proteínas imunorreativas foram detectadas. Duas proteínas apresentaram maior abundancia, com peso molecular, de 200 e 130 kDa respectivamente. Através da comparação de mapas proteômicos existentes e pelo peso molecular que estas proteínas apresentaram, sugere-se que as duas proteínas detectadas representem rOmpA (200 kDa) e rOmpB $(130 \mathrm{kDa})$. As demais proteínas detectadas apresentaram menor ocorrência e peso molecular inferior a $78 \mathrm{kDa}$, podendo representar membros da família de antígenos de superfície celular (Sca - Surface cell antigen). As proteínas detectadas poderão servir como base de estudo na elabo- 
ração de métodos diagnósticos sensíveis e específicos, no desenvolvimento de vacinas, além de possibilitarem novos estudos para terapias mais eficazes.

TERMOS DE INDEXAÇ̃̃O: Proteínas imunorreativas, Rickettsia sp. cepa Mata Atlântica, riquetsiose, bactéria intracelular, Febre Maculosa Brasileira, proteínas imunogênicas.

\section{INTRODUÇÃO}

A Febre Maculosa Brasileira (FMB) é uma doença zoonótica febril aguda, que tem como agente etiológico as bactérias do gênero Rickettsia. As riquétsias são bactérias da ordem Rickettsiales, família Rickettsiaceae, subgrupo $\alpha$-Proteobacteria e caracterizam-se por serem gram-negativas, intracelulares obrigatórias, cocobaciliar pleomórficas, visíveis à microscopia óptica comum (Raoult \& Roux 1997).

As diferentes espécies pertencentes ao gênero Rickettsia são classificadas em cinco grupos distintos: Grupo do Tifo (Thyphus Group - TG), Grupo de Transição (Transitional Group - TRG), Grupo Canadensis (Canadensis group - CG), Grupo Belli (Belli Group - BG) e Grupo da Febre Maculosa (Spotted Fever Group - SFG), sendo este último composto por mais de 22 espécies e subespécies (Weinert et al. 2009). Atualmente no Brasil, cinco espécies circulantes foram identificadas: Rickettsia rickettsii, Rickettsia riphicephali, Rickettsia amblyommi, Rickettsia felis e Rickettsia parkeri. No entanto, no Brasil, as principais espécies relacionadas aos sinais clínicos em humanos foram $R$. rickettsii e R. parkeri (Labruna et al. 2001). Estudos no Estado do Rio Grande do Sul descreveram R. parkeri como principal agente causador da doença (Sangioni et al. 2011).

R. parkeri, foi isolada pela primeira vez em Amblyomma maculatum, na Costa do Golfo dos Estados Unidos da América em 1937, porém foi considerada não patogênica (Parker et al. 1939). Apenas em 2004 foi classificada como responsável por causar Febre Maculosa em humanos, sendo considerada agente de uma leve ou moderada doença em muitos países do continente Americano (Paddock et al. 2004). Uma nova riquetsiose humana foi descrita como causadora de FMB no Estado de São Paulo, apresentando um quadro clínico semelhante em infecções causadas por R. parkeri (Spolidorio et al. 2010). Este novo agente riquetsial foi denominado de Rickettsia sp. cepa Mata Atlântica devido a sua similaridade filogenética com as espécies $R$. parkeri, Rickettsia africae e Rickettsia sibirica (Spolidorio et al. 2010). Além do Brasil, este novo agente foi identificado em outros países da América Latina, como Colômbia e Argentina, e na América Central (Lopes et al. 2016, Parola et al. 2013, Londoño et al. 2014, Monje et al. 2015). Estudos epidemiológicos demonstraram que as espécies envolvidas na transmissão do agente são Rhipicephalus sanguineus, Amblyomma aureolatum e Amblyomma ovale, sendo esta última considerada o principal vetor envolvido na disseminação de Rickettsia sp. cepa Mata Atlântica no Estado de São Paulo (Medeiros et al. 2011, Szabó et al. 2013).

Devido a sua natureza intracelular obrigatória, a manipulação genética das bactérias pertencentes ao gênero Rickettsia tem sido dificultada. Alternativamente, os perfis de expressão de proteínas (proteoma) têm sido utilizados para identificar os mecanismos de patogênese e diferenciar espécies riquétsiais reconhecendo a especificidade da resposta imune do hospedeiro frente às moléculas de superfície celular, referidas como proteínas de membrana externa (OMP) (Anacker et al. 1987). Mapas proteômicos tem sido o foco de diversas pesquisas envolvendo principalmente $R$. parkeri, Rickettsia prowazekii, Rickettsia conorii e R. felis. (Chao et al. 2004, Renesto et al. 2005a, Ogawa et al. 2007, Pornwiroon et al. 2009). Além das proteínas de membrana conhecidas (rOmpA e rOmpB) novas proteínas envolvidas na tradução e no metabolismo têm sido identificadas (Renesto et al. 2005a). Diante disso, este estudo teve como objetivo detectar e identificar proteínas desta nova cepa descrita, com o potencial de estimular o sistema imune do hospedeiro.

\section{MATERIAL E MÉTODOS}

Infecção experimental. $O$ antígeno foi obtido a partir de células VERO (green monkey kidney cells) infectadas com Rickettsia sp. cepa Mata Atlântica, mantidas em meio de cultivo (Dulbecco's Modified Eagle Medium ${ }^{\circledR}$ - DMEM, Life Technologies Corporation) com soro fetal bovino a $10 \%$, cultivadas em garrafas de cultivo celular de $50 \mathrm{~mL}$ e incubadas em estufa microbiológica a $28^{\circ} \mathrm{C}$, sendo realizadas três passagens em monocamadas celulares. 0 nível de infecção celular foi monitorado através da identificação do agente por meio da coloração de Gimenez, nas células infectadas e desprendidas da monocamada inoculada. A partir da última passagem, o tapete celular infectado foi desprendido através de um scraper e, juntamente com o meio de cultivo, foi transferido para tubos Falcon ${ }^{\circledR}$ estéreis com capacidade para $50 \mathrm{~mL}$, na concentração final de 2,5 x $10^{5}$ células VERO infectadas $/ \mathrm{mL}$.

Para obtenção de soro hiperimune, utilizou-se um coelho doméstico (Oryctolagus cuniculus), do sexo masculino, com idade de aproximadamente 90 dias, com peso inicial de 2,0 kg, sem contato prévio com carrapatos e produtos acaricidas. 0 animal foi inocu-

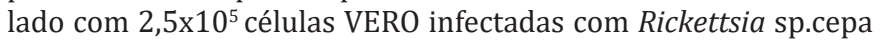
Mata Atlântica via intraperitoneal. Após 21 dias de infecção, foi coletado o sangue pela veia auricular e posteriormente obtido o soro sanguíneo. A amostra foi aliquotada, identificada e armazenada a $-70^{\circ} \mathrm{C}$ até o momento de uso.

Reação de imunofluorescência indireta (IFI). A amostra de soro sanguíneo foi submetida à IFI, a partir de lâminas sensibilizadas com antígenos brutos da cepa Mata Atlântica, conforme a descrição de Horta et al. (2004), para a confirmação da presença de anticorpos contra Rickettsia sp. O soro sanguíneo foi diluído a partir de 1:64 em Tampão salina fosfato (PBS) ( $\mathrm{NaCl} 8 \mathrm{~g} / \mathrm{L}, \mathrm{KCl}$ $0,2 \mathrm{~g} / \mathrm{L}, \mathrm{Na}_{2} \mathrm{HPO}_{4} 1,44 \mathrm{~g} / \mathrm{L}, \mathrm{KH}_{2} \mathrm{PO}_{4} 0,24 \mathrm{~g} / \mathrm{L}$ ). Em seguida, $15 \mu \mathrm{L}$ do material diluído foi depositado sobre cada poço das lâminas de IFI e incubados em câmara úmida a $37^{\circ} \mathrm{C}$ por 30 minutos. As lâminas foram lavadas com solução de Tampão de Lavagem $(\mathrm{NaCl}$ $8 \mathrm{~g} / \mathrm{L}, \mathrm{KCl}$ 0,2g/L, $\mathrm{Na}_{2} \mathrm{HPO}_{4}$ 1,44g/L, $\mathrm{KH}_{2} \mathrm{PO}_{4}$ 0,24g/L, Triton ${ }^{\circledR}$ $\mathrm{X}-100 \mathrm{~g} / \mathrm{L}$ ) e mantidas em cuba com a mesma solução durante 10 minutos, por duas vezes. Após a secagem, foram incubadas a $37^{\circ} \mathrm{C}$ por 30 minutos com isotiocianato de fluoresceína conjugado com imunoglobulina G anti-coelho (Sigma ${ }^{\circledR}$ ) na diluição de 1:300. Posteriormente foram novamente lavadas e coradas em câmara escura, em solução de Tampão de Lavagem e corante azul de Evans a $0,2 \%$. Após as lâminas estarem completamente secas, foram cobertas com glicerina tamponada e inserida a lamínula para realizar a leitura em microscópio com luz ultravioleta (Olympus BX60, Japan) com objetiva de 40x.

Extração das proteínas totais. A extração das proteínas totais foi realizada através da utilização do tampão Radio-Immuno- 
precipitation Assay (RIPA Buffer - Sigma ${ }^{\circledR}$ ) seguindo as recomendações do fabricante. As células VERO infectadas com Rickettsia sp.cepa Mata Atlântica foram removidas por centrifugação a $450 \mathrm{x}$ g por 5 minutos. 0 meio suspendido foi cuidadosamente retirado por aspiração. 0 pellet de células foi ressuspendido e lavado duas vezes com $1 \mathrm{~mL}$ de PBS. Centrifugou-se a 450 xg por 5 minutos e o meio suspendido foi retirado. Posteriormente foi adicionado $300 \mu \mathrm{L}$ de RIPA Buffer, seguido de homogeneização em um agitador para tubos e a solução armazenada a $2^{\circ} \mathrm{C}$ por 5 minutos. $\mathrm{O}$ lisado foi centrifugado a $8000 \mathrm{x}$ g por 10 minutos a $4^{\circ} \mathrm{C}$ para sedimentar os detritos celulares. 0 sobrenadante contendo a solução proteica foi cuidadosamente transferido para um microtubo, onde foi adicionado $3 \mu \mathrm{L}$ de coquetel inibidor de protease (Sig$\mathrm{ma}^{\circledR}$ ) e armazenado a $-20^{\circ} \mathrm{C}$ até o momento de uso.

SDS-PAGE. Os extratos proteicos foram homogeneizados com igual volume de tampão de amostra (Tris- $\mathrm{HCl} 0,065 \mathrm{M}$, SDS $2 \%$, glicerol $10 \%$, azul de bromofenol $0,0005 \%$ e $\beta$-mercaptoetanol $2,5 \%$ ) e aquecidos a $90^{\circ} \mathrm{C}$ por 4 minutos. As amostras preparadas foram centrifugadas a $14.000 x \mathrm{x}$ e submetidas à eletroforese em gel de poliacrilamida a $12 \%$ para o gel de separação e 7,5\% para o gel de aplicação, sob $120 \mathrm{~V}$ por 90 minutos em tampão Tris-glicina

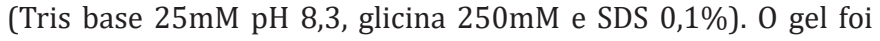
preparado com solução de acrilamida 30\%, SDS 10\%, APS 10\%, TEMED $(0,1 \%)$ e 1,5M de Tris $\mathrm{HCl}$ com $\mathrm{pH}$ de 8,8 para preparação do gel de separação e 1,5M de Tris $\mathrm{HCl}$ com pH de 6,8 para preparação do gel de aplicação. Posteriormente, foi corado com azul de Comassie em temperatura ambiente por 2 horas e lavado em solução descorante total (metanol 30\% e ácido acético 10\%) por 8 horas. 0 padrão das bandas de proteína foi analisado através da comparação com um marcador de massa molecular.

Western-blot. Após o fracionamento das proteínas por SDS-PAGE, realizou-se a análise da imunorreatividade das proteínas totais de Rickettsia sp. cepa Mata Atlântica através da técnica de Western-blot. As bandas de proteínas foram transferidas para membranas de nitrocelulose e, posteriormente, submetidas à detecção através de incubação com anticorpos primário e secundário.

0 gel (SDS-PAGE) contendo as proteínas fracionadas de $R i$ ckettsia sp.cepa Mata Atlântica e células VERO, foi transferido para a membrana de nitrocelulose, através do sistema de eletrotransferência úmida, onde permaneceu por 1 hora sob gradiente elétrico $25 \mathrm{~V}$. Ao término deste processo a membrana foi imersa em solução salina tamponada com 0,05 \% de Tween-20 (PBS-T) e $10 \%$ de leite em pó desnatado, para bloquear sítios de ligação inespecíficos. Após este procedimento, a membrana foi incubada com o soro do coelho previamente imunizados contra Rickettsia sp. cepa Mata Atlântica (anticorpo primário). 0 soro foi diluído em PBS-T e a membrana foi incubada por 1 hora a $37^{\circ} \mathrm{C}$, sob leve movimentação em agitador mecânico. Após, foi realizada duas lavagens da membrana em PBS-T. Posteriormente, a membrana foi incubada por mais $1 \mathrm{~h}$, com anticorpo anti-IgG espécie específico conjugado com peroxidase (anticorpo secundário - Anti-Rabbit IgG HRP Conjugate PROMEGA ${ }^{\circledR}$ ), para detectar os anticorpos primários ligados às proteínas. A revelação foi realizada através de substrato cromogênico. Devido a natureza intracelular do parasito e a dificuldade de extração de proteínas puras de Rickettsia sp. cepa Mata Atlântica, células VERO não infectadas foram utilizadas como controle na técnica de Western-blot, sendo incubadas com soro de coelho previamente imunizados contra Rickettsia sp. cepa Mata Atlântica nas mesmas condições para comparação com o extrato proteico da bactéria e assim garantir a detecção de proteínas imunorreativas exclusivas para esta nova espécie.

Comitê de Ética e Biossegurança. 0 projeto foi aprovado na Comissão de Ética no Uso de Animais (CEUA) da Universidade de São Paulo (USP), sob o número 2191/2011.

\section{RESULTADOS}

Após a inoculação experimental de Rickettsia sp. cepa Mata Atlântica $\left(2,5 \times 10^{5}\right.$ células infectadas $\left./ \mathrm{mL}\right)$ em coelho doméstico via intraperitoneal, observou-se títulos de até 1024 aos 21 dias pós infecção. Em relação a detecção de proteínas imunorreativas neste estudo, foram identificadas sete proteínas imunorreativas de Rickettsia sp. cepa Mata Atlântica, das quais 2 apresentaram alto peso molecular (cerca de 200 e $130 \mathrm{kDa}$ ) e 5 proteínas com menos de 78 kDa. Através da comparação de mapas proteômicos existentes e pela análise do peso molecular que estas duas proteínas de alto peso molecular apresentaram, sugere-se que rOmpA (200 kDa) e r0mpB (130 kDa) estejam entre as proteínas detectadas, e as demais proteínas identificadas possam pertencer a classe Sca - Surface cell antigen.

\section{DISCUSSÃO}

A membrana externa de uma bactéria é responsável pela ligação entre o microrganismo e o hospedeiro, desempenhando um papel essencial na adesão à célula e na evasão da resposta imune do hospedeiro (Mavromatis et al. 2006). As proteínas bacterianas que intercedem essas interações entre as diferentes espécies de riquétsias são alvos da resposta imune do hospedeiro e têm grande relevância no diagnóstico diferencial entre as espécies de riquétsias (Tjalsma et al. 2008).

Embora $R$. parkeri tenha sido identificada há mais de 70 anos, há relativamente poucos dados que descrevem a biologia, ou que identifiquem os componentes moleculares envolvidos no seu comportamento patogênico nos diferentes hospedeiros. Pornwiroon et al. (2009) construíram um mapa proteômico de referência para $R$. parkeri cepa Portsmouth através das técnicas de eletroforese bidimensional (2D) associada a espectrometria de massa por Dessorção e Ionização com Laser assistida por Matriz-Tempo-de-voo (MALDI-TOF), onde identificaram e caracterizaram 91 proteínas, de peso molecular variando de 10 a $240 \mathrm{kDa}$. No presente trabalho optou-se pela utilização da técnica de eletroforese em gel de poliacrilamida na presença de dodecil-sulfato de sódio (SDS-PAGE) para fracionamento do extrato proteico total de Rickettsia sp. cepa Mata Atlântica. Apesar de não haver diferenças significativas entre as técnicas de 2D e SDS-PAGE, esta última favorece a identificação de proteínas de baixo peso molecular (Ogawa et al. 2007).

SDS-PAGE é um método de eletroforese em gel para separar proteínas com base na sua massa. Essa técnica foi descrita por Laemmli (1970), quando a utilizou com a finalidade de determinar a mobilidade das proteínas em gel submetido à corrente elétrica. As proteínas são dissolvidas em dodecil sulfato de sódio (SDS), um detergente que remove as estruturas secundárias e terciárias da proteína e as mantêm como cadeia polipeptídica (Roy \& Kumar 2014). As proteínas menores se movem através do gel mais rapidamente, enquanto as moléculas maiores levam mais tempo de mobilidade, resultando em bandas localizadas no topo do gel.

A Identificação de proteínas de superfície celular expressas por bactérias do gênero Rickettsia é de grande interesse, pois servem como base para determinar antígenos 
imunorreativos com potencial para elaboração de vacinas, empregos em diagnósticos sensíveis e específicos, além de possibilitarem novos estudos para terapias mais eficazes (Tjalsma et al. 2008). Bactérias do gênero Rickettsia apresentam diferentes proteínas pertencentes a várias categorias funcionais, incluindo a replicação (SSB), transcrição (RPOA e PnP) e tradução (SERS, Gata, GATB, EF-Ts, EF-Tu, HtrA, e Pepa), além de outras diferentes proteínas envolvidas no metabolismo da energia (Renesto et al. 2005a). Bactérias intracelulares, tais como riquétsias têm um sistema metabólico limitado, que é compensada pelo transporte através da membrana de fontes de energia a partir do ambiente (Renesto et al. 2005b). Ogawa et al. (2007) detectaram um número limitado de proteínas envolvidas em funções metabólicas e vários componentes do transportador ABC em R. felis. Além disso, observaram que as principais proteínas obtidas são aquelas envolvidas com a tradução, principalmente proteínas ribossomais, caracterizando um padrão de abundância em bactérias intracelulares obrigatórias, quando comparado com outros grupos bacterianos.

As células riquétsiais são compostas por uma camada proteica cristalina, denominada de S-camada, a qual é composta por antígenos proteicos de superfície imunodominantes (SPAs) (Palmer et al. 1974, Dasch 1981). As duas principais proteínas de superfície imunodominante presentes em diversas espécies de riquétsias são rOmpA e rOmpB, ambas estão envolvidas na adesão da bactéria à célula hospedeira (Anacker et al. 1987, Xu \& Raoult 1997). Ambos os antígenos foram identificados em diferentes espécies de riquétsias e são os principais determinantes antigênicos responsáveis por desencadear uma resposta imunitária em pacientes infectados (Teysseire \& Raoult 1992). rOmpA e rOmpB são duas proteínas grandes, com cerca de 190 kDa e 135 kDa de peso molecular, respectivamente e não apresentam semelhança de sequência significativa, exceto o fato de ambas exibirem uma região altamente conservada (Gilmore et al. 1991, Li \& Walker 1998). Esta região, identificada como auto transportadora (AT), possui estruturas modulares considerados fatores de virulência, incluindo uma sequência de sinal N-terminal, um peptídeo central de passageiro e um de translocação C-terminal ( $\beta$-péptido) (Jacob-Dubuisson et al. 2004). Tanto rOmpA quanto rOmpB têm demonstrado mediar a ligação do parasito às células hospedeiras, comprovando assim o seu grande potencial imunorreativo e a alta capacidade em induzir a imunidade protetora no hospedeiro (Díaz-Montero et al. 2001, Feng et al. 2004a, 2004b).

A Febre Maculosa pode ser diagnosticada através de numerosos processos sorológicos, mas a IFI é considerada como padrão-ouro para a detecção do antígeno, sendo descrita como a técnica mais conveniente e sensível (La Scola \& Raoult 1997). No entanto, a especificidade de todas as técnicas de diagnóstico sorológico é limitada devido às reações cruzadas que apresentam entre as espécies de riquétsias. Sendo assim, esta reatividade varia conforme a técnica utilizada e o animal hospedeiro a partir do qual o soro anti-Rickettsia é obtido (Philip et al. 1978). Os antígenos lipopolissacarideos (LPS) reagem de forma cruzada entre as riquétsias do SFG e TG, além de reagirem com ou- tras espécies bacterianas, como Proteus spp. e Legionella spp. (Sompolinsky et al. 1986). Vários trabalhos têm estudado a identificação de proteínas heterólogas entre as espécies pertencentes ao gênero Rickettsia. Pornwiroon et al. (2009) identificaram novas proteínas imunorreativas para $R$. parkeri, entre elas reconhecidas por soro de pacientes humanos, caracterizando-as como possíveis alvos potenciais para o diagnóstico e prevenção da doença. Qi et al. (2013) identificaram proteínas de superfície expostas (SEPs) em Rickettsia heilongjiangensis, através de soro de camundongos infectados, onde OmpA-2 e GroEL obtiveram maior sororreatividade, demonstrando assim o potencial para o desenvolvimento de testes sorológicos eficazes.

Tanto a resposta imune humoral, quanto a celular modulam a infecção por riquétsias. Após a recuperação do hospedeiro, que ocorre em torno de 15 dias pós-infecção, a imunidade é sólida e duradoura, não havendo relatos de reinfecções em humanos ou animais. No entanto, os mecanismos de imunidade protetora não estão completamente elucidados (Valbuena et al. 2002).

Atualmente os principais candidatos para o desenvolvimento de uma vacina eficaz são as proteínas rOmpA e rOm$\mathrm{pB}$, pois induzem resposta imune mediada por células T e humorais (Anacker et al. 1987, Sumner et al. 1995). Díaz-Montero et al. (2001) observaram a imunização de camundongos frente a uma infecção experimental de $R$. conorii ao aplicarem uma vacina a partir de fragmentos dos genes de rOmpA e rOmpB. Os dados validam ambas as proteínas como imunogênicas potentes e, portanto, como fontes de elementos imuno-estimuladores com especificidade para os linfócitos T, que são os principais responsáveis pela imunidade protetora efetiva contra infecções por riquétsias.

Estes resultados foram comprovados por outros estudos em diferentes espécies de riquétsias em diferentes hospedeiros. Crocquet-Valdes et al. (2001) demonstraram o potencial imunoestimulador da proteína rOmpA ao utiliza-la como vacina recombinante em camundongos, onde os mesmos apresentaram uma significativa resposta imune ao serem desafiados com $R$. conorii. De maneira semelhante, Sumner et al. (1995) inocularam uma proteína recombinante de rOmpA em cobaias, os quais desenvolveram anticorpos reativos contra $R$. rickettsii. Para as bactérias pertencentes ao SFG, a proteína $\mathrm{rOmpA}$ representa o principal antígeno candidato para produção de vacinas, devido esta proteína apresentar um papel importante na adesão inicial de $R$. rickettsii às células hospedeiras (Li \& Walker 1998).

Outros cinco antígenos detectados de peso molecular inferior a $78 \mathrm{kDa}$ possivelmente pertençam a família de antígenos de superfície celular (Sca - Surface cell antigen). As proteínas membros da família Sca desempenham papéis importantes na ligação à célula hospedeira e têm sido propostos como bons fragmentos para o desenvolvimento de vacinas (Renesto et al. 2005a, Blanc et al. 2005). Renesto et al. (2005a) identificaram uma espécie de proteína imunorreagente com $60 \mathrm{kDa}$ de peso molecular. 0 reconhecimento se deu tanto para soros positivos para $R$. conorii de pacientes humanos, como também de animais de laboratório. A proteína imunodetectada foi identificada como GroEL, através de espectrometria de massa. 
GroEL é uma proteína de choque térmico que tem demonstrado estar localizado nas superfícies de diversas bactérias rickettsiais (Renesto et al. 2005a, Ogawa et al. 2007, Pornwiroon et al. 2009, Sears et al. 2012). Proteínas de choque térmico são componentes essenciais distribuídos por toda a superfície bacteriana e tem como função garantir que as proteínas do genoma codificado sejam expressas como moléculas funcionais (Hartl 1991). O papel destas proteínas evolutivamente conservadas é importante em condições adversas, pois impedem o enrolamento incorreto e a agregação da proteína (Fayet et al. 1989). As bactérias do gênero Rickettsia são transmitidas ao homem por carrapatos, e sua sobrevivência requer adaptação a diferentes ambientes, incluindo mudanças fisiológicas e nutricionais entre os diferentes hospedeiros. Renesto et al. (2005a) comprovaram essas mudanças metabólicas necessárias a sobrevivência da bactéria ao detectar proteínas de choque térmico em alta quantidade em $R$. conorii, sugerindo que este fato esteja correlacionado com a exigência metabólica da bactéria em resistir a condições adversas, sendo muitas delas letais para o patógeno.

Homólogos putativos GroEL foram detectados e identificados em diferentes espécies de riquétsias: Rickettsia akarii, Rickettsia australis, Rickettsia bellii e Rickettsia rickettsii (Eremeeva et al. 1993). Radulovic et al. (2002) compararam a sequência de aminoácidos de GroEL de Rickettsia typhi com R. conorii e R. prowazekii fornecendo fortes evidências de semelhanças entre as espécies. Apesar do elevado grau de conservação de aminoácidos, esta proteína tem demonstrado ser uma promissora candidata para elaboração de vacina contra várias infecções microbianas, como Mycobacterium tuberculosis e Helicobacter pylori (Mustafa 2002, Yamaguchi et al. 2003).

Pornwiroon et al. (2009) identificaram outras três proteínas com potencial imunológico ao analisar $R$ parkeri cepa Portsmouth: fator de início de tradução (IF-2), proteína de divisão celular (FtsZ), e cisteína-tRNAsintetase. As duas últimas apresentam baixo peso molecular, ambas apresentando em torno de $50 \mathrm{kDa}$. Todas as proteínas até então não têm sido descritas para outras riquétsias, sugerindo que representam proteínas imunologicamente reativas exclusivas de $R$. parkeri. No entanto, a antigenicidade de IF-2 e FtsZ tem sido relatado em outros trabalhos com diferentes espécies bacterianas de diferentes gêneros (Boonjakuakul et al. 2007, Zhang et al. 2008).

\section{CONCLUSÕES}

Foram detectadas sete proteínas imunorreativas.

Através da comparação dos mapas proteômicos existentes e por meio do peso molecular que estas proteínas apresentaram, sugere-se que rOmpA (200 kDa) e rOmpB $(130 \mathrm{kDa})$ estejam entre as proteínas imunorreativas dominantes detectadas.

Outras cinco proteínas foram identificadas, todas apresentando peso molecular inferior a $78 \mathrm{kDa}$, sendo sugestivo que pertençam a classe Sca - Surface cell antigen. Contudo, estudos empregando a técnica de Cromatografia Líquida Moderna associada a Espectrometria de Massas (LC/MS/ MS) deverão ser conduzidos para caracterização proteica.
Além disso, outros estudos são necessários, a fim de determinar se as proteínas detectadas representam proteínas imunologicamente reativas únicas para Rickettsia sp. cepa Mata Atlântica, ou se são homólogas a outras espécies.

Os resultados preliminares obtidos nesse estudo representaram uma primeira etapa para a busca de proteínas alvo capazes de proporcionar o desenvolvimento de testes de diagnósticos ainda mais específicos e poderá subsidiar estudos para o desenvolvimento de vacinas, bem como possibilitar a análise proteomica estrutural e funcional de Rickettsia, podendo ser útil para avaliar diferenças na capacidade das diferentes espécies em estimular a resposta imunológica do hospedeiro.

\section{REFERÊNCIAS}

Anacker R.L., MannR.E. \& Gonzales C. 1987. Reactivity of monoclonal antibodies to Rickettsia rickettsia with spotted fever and typhus group rickettsiae. J. Clin. Microbiol. 25:167-171.

Blanc G., Ngwamidiba M., Ogata H., Fournier P.E., Claverie J.M. \& Raoult D. 2005. Molecular evolution of Rickettsia surface antigens: evidence of positive selection. Mol. Biol. Evol. 22(10):2073-2083.

Boonjakuakul J.K., Gerns H.L., Chen Y.T., Hicks L.D., Minnick M.F., Dixon S.E., Hall S.C. \& Koehler J.E. 2007. Proteomic and immunoblot analyses of Bartonella quintana total membrane proteins identify antigens recognized by sera from infected patients. Infect. Immun. 75(5):25482561.

Chao C.C., Chelius D., Zhang T., Daggle L. \& Ching W.M. 2004. Proteome analysis of Madrid E strain of Rickettsia prowazekii. Proteomics 4(5):1280-1292.

Crocquet-Valdes P.A., Díaz-Montero C.M., Feng H.M., Li H., Barrett A.D.T. \& Walker D.H. 2001. Immunization with a portion of rickettsial outer membrane protein A stimulates protective immunity against spotted fever rickettsiosis. Vaccine 20(5/6):979-988.

Dasch G.A. 1981. Isolation of species-specific protein antigens of Rickettsia typhi and Rickettsia prowazekii for immunodiagnosis and immunoprophylaxis. J. Clin. Microbiol. 14(3):333-341.

Díaz-Montero C.M., Feng H.M., Crocquet-Valdes P.A. \& Walker D.H. 2001. Identification of protective components of two major outer membrane proteins of spotted fever group rickettsiae. Am. J. Trop. Med. Hyg. 65(4):371-378.

Eremeeva M.E., Balayeva N.M., Ignatovich V.F. \& Raoult D. 1993. Proteinic and genomic identification of spotted fever group rickettsiae isolated in the former USSR. J. Clin. Microbiol. 31(10):2625-2633.

Fayet O., Ziegelhoffer T. \& Georgopoulos C. 1989. The groES and groEL heat shock gene products of Escherichia coli are essential for bacterial growth at all temperatures. J. Bacteriol. 171(3):1379-1385.

Feng H.M., Whitworth T., Olano J.P., Popov V.L. \& Walker D.H. 2004 a. Fc-dependent polyclonal antibodies and antibodies to outer membrane proteins A and B, but not to lipopolysaccharide, protect SCID mice against fatal Rickettsia conorii infection. Infect. Immun. 72(4):2222-2228.

Feng H.M., Whitworth T., Popov V. \& Walker D.H. 2004b. Effect of antibody on the rickettsia-host cell interaction. Infect. Immun. 72(6):35243530.

Gilmore Jr R.D., Cieplak Jr W., Policastro P.F. \& Hackstadt T. 1991. The 120 kilodalton outer membrane protein (rOmp B) of Rickettsia rickettsii is encoded by an unusually long open reading frame: evidence for protein processing from a large precursor. Mol. Microbiol. 5(10):2361-2370.

Hartl F.U. 1991. Heat shock proteins in protein folding and membrane translocation. Semin. Immunol. 3(1):5-16.

Horta M.C., Labruna M.B., Sangioni L.A., Vianna M.C.B., Gennari S.M., Galvão M.A.M., Mafra C.L., Vidotto O., Schumaker T.T.S. \& Walker D.H. 2004. Prevalence of antibodies to spotted fever group rickettsiae in humans and domestic animals in a Brazilian spotted fever-endemic area in the state of São Paulo, Brazil: serologic evidence for infection by Rickettsia 
rickettsii and another spotted fever group Rickettsia. Am. J. Trop. Med. Hyg. 71(1):93-97.

Jacob-Dubuisson F., Fernandez R. \& Coutte L. 2004. Protein secretion through autotransporter and two-partner pathways. Biochim. Biophys. Acta 1694(1/3):235-257.

La Scola B. \& Raoult D. 1997. Laboratory diagnosis of Rickettsioses: current approaches to diagnosis of old and new rickettsial diseases. J. Clin. Microbiol. 35(11):2715-2727.

Labruna M.B., Mattar V.S., Nava S., Bermudez S., Venzal J.M., Dolz G., Abarca K., Romero L., Sousa R., Oteo J. \& Zavala-Castro J. 2001. Rickettsioses in Latin America, Caribbean, Spain and Portugal. Revta MVZ Cordoba 16(2):2435-2457.

Laemmli U.K. 1970. Cleavage of structural proteins during the assembly of the head of bacteriophage T4. Nature 227(5259):680-685

Li H. \& Walker D.H. 1998. rOmpA is a critical protein for the adhesion of Rickettsia rickettsia to host cells. Microb. Pathog. 24(5):289-298.

Londoño A.F., Díaz F.J., Valbuena G., Gazi M., Labruna M.B., Hidalgo M., Mattar S., Contreras V. \& Rodas J.D. 2014. Infection of Amblyomma ovale by Rickettsia sp. strain Atlantic rainforest, Colombia. Ticks Tick Borne Dis. 5(6):672-675.

Lopes M.G., Junior J.M., Foster R.J., Harmsen B.J., Sanchez E., Martins T.F., Quigley H., Marcili A. \& Labruna M.B. 2016. Ticks and rickettsiae from wildlife in Beliza, Central America. Parasit. Vectors 9(62):1-7.

Mavromatis K., Doyle C.K., Lykidis A., Ivanova N., Francino M.P., Chain P., Shin M., Malfatti S., Larimer F., Copeland A., Detter J.C., Land M., Richardson P.M., Yu X.J., Walker D.H., McBride J.W. \& Kyrpides N.C. 2006. The genome of the obligately intracellular bacterium Ehrlichia canis reveals theme as of complex membrane structure and immune evasion strategies. J. Bacteriol. 188(11):4015-4023.

Medeiros A.P., Souza A.P., Moura A.B., Lavina M.S., Bellato V., Sartor A.A., Nieri-Bastos F.A., Richtzenhain L.J. \& Labruna M.B. 2011. Spotted fever group Rickettsia infecting ticks (Acari: Ixodidae) in the state of Santa Catarina, Brazil. Mem. Inst. Oswaldo Cruz 106(8):926-930.

Monje L.D., Nava S., Eberhardt A.T., Correa A.I., Guglielmone A.A. \& Beldomenico P.M. 2015. Molecular detection of the human pathogenic Rickettsia sp. strain Atlantic rainforest in Amblyomma dubitatum ticks from Argentina. Vector Borne Zoonotic Dis. 15(2):167-169.

Mustafa A.S. 2002. Development of new vaccines and diagnostic reagents against tuberculosis. Mol. Immun. 39(1/2):113-119.

Ogawa M., Renesto P., Azza S., Moinier D., Fourquet P., Gorvel J.P. \& Raoult D. 2007. Proteome analysis of Rickettsia felis highlights the expression profile of intracellular bacteria. Proteomics 7(8):1232-1248.

Paddock C.D., Sumner J.W., Comer J.A., Zaki S.R., Goldsmith C.S., Goddard J., McLellan S.L.F., Tamminga C.L. \& Ohl C.A. 2004. Rickettsia parkeri: a newly recognized cause of spotted fever rickettsiosis in the United States. Clin. Infect. Dis. 38(6):805-811.

Palmer E.L., Martin M.L. \& Mallavia L. 1974. Ultrastucture of the surface of Rickettsia prowazek and Rickettsia akari. Appl. Environ. Microbiol. 28(4):713-716.

Parker R.R., Kohls G.M., Cox G.W. \& Davis G.E. 1939. Observations of an infectious agent from Amblyomma maculatum. Publ. Health Rep. 54(32):1482-1484.

Parola P., Paddock C.D., Socolovschi C., Labruna M.B., Mediannikov O., Kernif T., Abdad M.Y., Stenos J., Bitam I., Fournier P.E. \& Raoult D. 2013. Update on tick-borne rickettsioses around the world: a geographic approach. Clin. Microbiol. Rev. 26(4):657-702.

Philip R.N., Casper E.A., Burgdorfer W., Gerloff R.K., Hughes L.E. \& Bell E.J. 1978. Serologic typing of rickettsiae of the spotted fever group by micro immunofluorescence. J. Immun. 121(5):1961-1968.

Pornwiroon W., Bourchookarn A., Paddock C.D. \& Macaluso K.R. 2009. Proteomic analysis of Rickettsia parkeri strain Portsmouth. Infect. Immun. 77(12):5262-5271.
Qi Y., Xiong X., Wang X., Duan C., Jia Y., Jiao J., Gong W. \& Wen B. 2013. Proteome analysis and serological characterization of surface-exposed proteins of Rickettsia heilongjiangensis. PLoS One 8(7):1-13.

Radulovic S., Rahman M.S., Beier M.S. \& Azad A.F. 2002. Molecular and functional analysis of the Rickettsia typhi groESL operon. Gene 298(1):41-48.

Raoult D. \& Roux V. 1997. Rickettsioses, as paradigms of new or emerging infectious diseases. Clin. Microbiol. Rev. 10(4):694-719.

Renesto P., Azza S., Dolla A., Fourquet P., Vestris G., Gorvel J.P. \& Raoult D. 2005a. Proteome analysis of Rickettsia conorii by two-dimensional gel electrophoresis coupled with mass spectrometry. FEMS Microbiol. Lett. 245(2):231-238.

Renesto P., Ogata H., Audic S., Claverie J.M. \& Raoult D. 2005b. Some lessons from Rickettsia genomics FEMS Microbiol. Rev. 29(1):99-117.

Roy S. \& Kumar V.A. 2014. Practical approach on SDS PAGE for separation of protein. Int. J. Sci. Res. 3(8):955-960.

Sangioni L.A., Vogel F.F.S., Cadore G.C., Hilger R.B., Tonim R., Pacheco R.C., Ogrzewalska M. \& Labruna M.B. 2011. Rickettsial infection in Cerro Largo, State of Rio Grande do Sul, Brazil. Arq. Bras. Med. Vet. Zootec. 63(2):511-514.

Sears K.T., Ceraul S.M., Gillespie J.J., Allen Jr E.D., Popov V.L., Ammerman N.C., Rahman M.S. \& Azad A.F. 2012. Surface proteome analysis and characterization of surface cell antigen (SCA) or autotransporter family of Rickettsia typhi. PLoS Pathog. 8(8):1-17.

Sompolinsky D., Boldur I., Goldwasser R.A., Kahana H., Kazak R., Keysary A. \& Pik A. 1986. Serological cross-reactions between Rickettsia typhi, Proteus vulgaris OX19, and Legionella bozemanii in a series of febrile patients. Isr. J. Med. Sci. 22(10):745-752.

Spolidorio M.G., Labruna M.B., Mantovani E., Brandão P.E., Richtzenhain L.J. \& Yoshinari N.H. 2010. Novel spotted fever group rickettsiosis, Brazil. Emerg. Infect. Dis. 16(3):521-523.

Sumner J.W., Sims K.G., Jones D.C. \& Anderson B.E. 1995. Protection of guinea-pigs from experimental Rocky Mountain spotted fever by immunization with baculovirus-expressed Rickettsia rickettsia rOmpA protein. Vaccine 13(1):29-35.

Szabó M.P.J., Nieri-Bastos F.A., Spolidorio M.G., Martins T.F., Barbieri A.M. \& Labruna M.B. 2013. In vitro isolation from Amblyomma ovale (Acari: Ixodidae) and ecological aspects of the Atlantic rainforest Rickettsia, the causative agent of a novel spotted fever rickettsiosis in Brazil. Parasitology 140(6):719-728.

Teysseire N. \& Raoult D. 1992. Comparison of western immunoblotting and microimmunofluorescence for diagnosis of Mediterranean spotted fever. J. Clin. Microbiol. 30(2):455-460.

Tjalsma H., Schaeps R.M.J. \& Swinkels D.W. 2008. Immunoproteomics: from biomarker discovery to diagnostic applications. Prot. Clin. Appl. 2(2):167-180.

Valbuena G., Feng H.M. \& Walker D.H. 2002. Mechanisms of immunity against rickettsiae: new perspectives and opportunities offered by unusual intracellular parasites. Microb. Infect. 4(6):625-633.

Weinert L.A., Werren J.H., Aebi A., Stone G.N. \& Jiggins F.M. 2009. Evolution and diversity of Rickettsia bacteria. BMC Biol. 7(6):1-15.

Xu W. \& Raoult D. 1997. Distribution of immunogenic epitopes on the two major immunodominant proteins (rOmpA and rOmpB) of Rickettsia conorii among the other rickettsiae of the spotted fever group. Clin. Vaccine Immun. 4(6):753-763.

Yamaguchi H., Osaki T., Taguchi H., Sato N., Toyoda A., Takahashi M., Kai M., Nakata N., Komatsu A., Atomi Y. \& Kamiya S. 2003. Effect of bacterial flora on post immunization gastritis following oral vaccination of mice with Helicobacter pylori heat shock protein 60. Clin. Vaccine Immun. 10(5):808-812.

Zhang A., Xie C., Chen H. \& Jin M. 2008. Identification of immunogenic cell wall-associated proteins of Streptococcus suis serotype 2. Proteomics 8(17):350-351. 